Bio - grafía. Escritos sobre la Biología y su Enseñanza. ISSN 2027-1034

Edición Extraordinaria. p.p. 1325 - 1332

Memorias del IX Encuentro Nacional de Experiencias en Enseñanza de la Biología y la Educación Ambiental. IV Congreso Nacional de Investigación en Enseñanza de la Biología.

\title{
REPRESENTACIONES DE LOS MAESTROS DE PRIMARIA EN UNA INSTITUCIÓN PÚBLICA DE BARRANCABERMEJA, SOBRE LANATURALEZA DE LA CIENCIA DESDE UNA NOCIÓN DOGMÁTICA Y CONSTRUCTIVISTA
}

\author{
REPRESENT ATIONS OF PRIMARY SCHOOL TEACHERS IN A PUBLIC \\ INSTITUTION OF BARRANCABERMEJA, ABOUT THE NATURE OF SCIENCE \\ FROM ADOGMATIC AND CONSTRUCTIVIST NOTION
}

\author{
Edna Rocio Pérez ${ }^{1}$ \\ José Rafael Arrieta Vergara ${ }^{2}$ \\ Silvio Fernando Daza Rosales ${ }^{3}$
}

\section{RESUMEN}

El presente trabajo de investigación, buscó determinar las representaciones de los profesores de primaria sobre la naturaleza de la ciencia ( $\mathrm{NdC})$ de una institución educativa pública en Barrancabermeja. Se planteó una investigación de enfoque cualitativo del tipo descriptivo. A 17 docentes se les aplicó un cuestionario para conocer su opinión sobre las características de una visión epistemológica específica, desde los enfoques: dogmático y constructivista. El análisis de los resultados mostró en los profesores una coexistencia de representaciones debido a la débil formación y falta de reflexión sobre la naturaleza del conocimiento científico. Predominan las representaciones desde un enfoque dogmático positivista con la fuerte creencia del método científico universal. Sus ideas ponen de manifiesto concepciones empiristas de la naturaleza de la ciencia asignándole un importante papel al carácter experimental de la ciencia, que influye en su manera de enseñar al considerarlas como un cuerpo de conocimientos irrefutables, verdaderos y definitivos. Existen indicios del enfoque constructivista cuando le dan valor a la intuición y la imaginación en el uso de la metodología científica y cuando reconocen que deben adoptar un modelo de ciencia y de enseñanza de la ciencia epistemológicamente fundamentada.

PALABRAS CLAVE: Naturaleza de la ciencia, representaciones, corrientes epistemológicas, enseñanza de la ciencia.

\section{ABSTRACT}

This research work, sought to determine the representations of primary school teachers about the nature of Science $(\mathrm{NdC})$ of a public educational institution in Barrancabermeja. A research of qualitative descriptive approach arose. 17 teachers them a questionnaire was applied to know your opinion about the characteristics of a specific epistemological vision, from approaches: constructivist and dogmatic. The

\footnotetext{
1 Instituto Universitario da Paz, UNIPAZ, Grupo de investigación en enseñanza de la ciencias y seguridad alimentaria, INYUBA, rocio_1718z@hotmail.com

2 Instituto Universitário da Paz, UNIPAZ, lider grupo de investigacion en enseñanza de la ciencias y seguriadad alimentaria, INYUBA, rafael.arrieta@unipaz.edu.co

3 Instituto Universitário da Paz, UNIPAZ, Grupo de investigacion en enseñanza de la ciencias y seguriadad alimentaria, INYUBA, silvio.daza@unipaz.edu.co
} 
Bio - grafía. Escritos sobre la Biología y su Enseñanza. ISSN 2027-1034

Edición Extraordinaria. p.p. 1325 - 1332

Memorias del IX Encuentro Nacional de Experiencias en Enseñanza de la Biología y la Educación Ambiental. IV Congreso Nacional de Investigación en Enseñanza de la Biología.

analysis of the results showed teachers a coexistence of representations due to weak training and lack of reflection on the nature of scientific knowledge. Dominated by representations from a positivist dogmatic approach with the strong belief of universal scientific method. Their ideas highlight conceptions empiricists of the nature of science assigning an important role to the experimental nature of the science, that influences their way of teaching to consider them as a body of irrefutable, true and definitive knowledge. There are indications of the constructivist approach when they give value to the intuition and imagination in the use of scientific methodology and when they recognize that they should adopt a model of science and epistemologically-based science teaching.

KEY WORDS: Nature of representations, epistemological currents, science, teaching science.

\section{INTRODUCCION}

Un elemento que puede incidir en la forma como se aborda la enseñanza de la ciencia, son las representaciones sobre la naturaleza de las ciencias de los docentes, quien genera concepciones precisas sobre la manera como un alumno aprende, sobre las finalidades de la enseñanza, sus metodologías y sistemas de evaluación. (Arrieta \& Daza, 2014). Estas opiniones y creencias que tienen acerca del conocimiento científico pueden representar verdaderos obstáculos en el desarrollo de su práctica docente (Porlan \& del Pozo, 1996).

El conocimiento sobre la naturaleza de la ciencia guía a los profesores para hacer una descripción adecuada a los estudiantes, por lo que encontrar su relación con el aprendizaje, puede aportar a la compresión de los procesos de enseñanza y es posible encontrar correspondencia entre las ideas de los profesores sobre la naturaleza de las ciencias, sus ideas sobre el conocimiento científico y didáctico y el curricular (Tamayo etal, 2010).

Las ideas del profesorado de ciencias acerca de la naturaleza de la ciencia pueden convertirse en obstáculos, cuando se quieren lograr aprendizajes, especialmente, en estudiantes con diferencias y por eso es muy importante el nivel de comprensión de los docentes en este tema (Pujalte etal, 2014). Aunque poseen una comprensión de cómo opera la ciencia, no la relacionan con la enseñanza de las mismas y pareciera ser que esta forma de abordar la enseñanza, podrían generar dificultades en los estudiantes, en su forma de pensar, de preguntar y de comportarse en el aula. lgualmente puede influir en el docente que podrían encontrar dificultades para desarrollar sus actividades, evaluar, plantear ejercicios, proponer innovaciones en los currículos, entre otros (Arrieta \& Daza, 2014).

Se evidencia la necesidad y pertinencia para identificar y caracterizar las representaciones acerca de las ciencias de los profesores en vista que éstas configuran el marco metateórico dentro del cual se desarrollan sus clases por lo que se propuso la siguiente pregunta ¿Cuáles son las representaciones sobre la naturaleza de las ciencias que tienen los docentes de nivel primario en una institución pública de Barrancabermeja? 
Bio - grafía. Escritos sobre la Biología y su Enseñanza. ISSN 2027-1034

Edición Extraordinaria. p.p. 1325 - 1332

Memorias del IX Encuentro Nacional de Experiencias en Enseñanza de la Biología y la Educación Ambiental. IV Congreso Nacional de Investigación en Enseñanza de la Biología.

\section{METODOLOGÍA}

El proyecto se desarrolló en la institución educativa Diego Hernández de Gallegos, Barrancabermeja, Santander, Colombia, Fue una investigación de enfoque cualitativo del tipo descriptivo. Participaron 17 profesoras con una experiencia entre 16-20 años y se desempeñan en su mayoría en las áreas de español, matemáticas y ciencias naturales. Para el análisis se utilizó la red conceptual propuesta por Wang y Marsh (2002) que considera tres niveles de comprensión docente: Conceptual, contextual y procedimental, igualmente y se tuvo en cuenta la red conceptual para la dimensión naturaleza de la ciencia propuesta por Camacho y Quintanilla citado por Wang y Marsh (2002). Se empleó un cuestionario de escala Likert acerca de la imagen de ciencia y educación científica de profesores. cuenta con 60 enunciados y las dimensiones naturaleza de la ciencia, enseñanza de las ciencias, historia de las ciencias, aprendizaje de las ciencias, evaluación de los aprendizajes científicos y rol del profesor (Quintanilla Et al, 2005). Para la investigación solo se consideró la dimensión Naturaleza de la ciencia, se tomaron siete enunciados del instrumento original sin afectar la imagen epistemológica que representa la dimensión naturaleza de la ciencia ni sus enfoques.

\section{RESULTADOS Y DISCUSIÓN}

La metodología científica permite al investigador en ciencias utilizar la intuición y la imaginación en cualquier momento del proceso de construcción científica. El $76 \%$ de los docentes están de acuerdo sobre el uso y la intuición en el proceso de construcción científica lo que coincide con Quintanilla y Ravanal (2009) quienes resaltan la importancia de estos en el proceso de investigación científica, ver cuadro 1.

\section{Cuadro 1:}

El uso de la intuición y la imaginación en el proceso de construcción científica.

\begin{tabular}{|c|c|c|}
\hline Valoración & Número & Porcentaje \\
\hline Totalmente de Acuerdo & 5 & $29 \%$ \\
\hline Parcialmente de Acuerdo & 8 & $47 \%$ \\
\hline Parcialmente en Desacuerdo & 2 & $12 \%$ \\
\hline Totalmente en Desacuerdo & 2 & $12 \%$ \\
\hline Total & 17 & $100 \%$ \\
\hline
\end{tabular}

Las ciencias tienen carácter experimental, porque es indispensable para construir los hechos científicos, a partir de los hechos del mundo. El 100\% de los docentes están de acuerdo con el carácter experimental de las ciencias y de su importancia para construir los hechos científicos, Cuadro 2. 
Bio - grafía. Escritos sobre la Biología y su Enseñanza. ISSN 2027-1034

Edición Extraordinaria. p.p. 1325 - 1332

Memorias del IX Encuentro Nacional de Experiencias en Enseñanza de la Biología y la Educación Ambiental. IV Congreso Nacional de Investigación en Enseñanza de la Biología.

\section{Cuadro 2}

Las ciencias tienen carácter experimental, porque es indispensable para construir los hechos científicos, a partir de los hechos del mundo.

\begin{tabular}{|c|c|c|}
\hline Valoración & Número & Porcentaje \\
\hline Totalmente de Acuerdo & 8 & $47 \%$ \\
\hline Parcialmente de Acuerdo & 9 & $53 \%$ \\
\hline Parcialmente en Desacuerdo & 0 & $0 \%$ \\
\hline Totalmente en Desacuerdo & 0 & $0 \%$ \\
\hline Total & 17 & $100 \%$ \\
\hline
\end{tabular}

Los criterios que poseen las ciencias son parciales porque los hechos de la naturaleza están sujetos a interpretaciones individuales y sociales. El $88 \%$ están de acuerdo sobre la importancia de las interpretaciones sociales e individuales sobre los hechos de la naturaleza y sobre la visión parcial de las ciencias y su carácter provisional del conocimiento (Acevedo, 2008), ver Cuadro 3.

Cuadro 3:

La parcialidad en los criterios de las ciencias y las interpretaciones individuales y sociales.

\begin{tabular}{|c|c|c|}
\hline Valoración & Número & Porcentaje \\
\hline Totalmente de Acuerdo & 5 & $29 \%$ \\
\hline Parcialmente de Acuerdo & 10 & $59 \%$ \\
\hline Parcialmente en Desacuerdo & 2 & $12 \%$ \\
\hline Totalmente en Desacuerdo & 0 & $0 \%$ \\
\hline & 17 & $100 \%$ \\
\hline
\end{tabular}

El profesor de ciencias debe adoptar un modelo de ciencia y de enseñanza fundamentado epistemológicamente. El 94\% de los encuestados están de acuerdo sobre la adopción de un modelo de ciencia y de su enseñanza bien fundamentado, reconocen la importancia de implementar nuevas estrategias y modelos de enseñanza en su labor docente, Cuadro 4.

Cuadro 4: 
Bio - grafía. Escritos sobre la Biología y su Enseñanza. ISSN 2027-1034

Edición Extraordinaria. p.p. 1325 - 1332

Memorias del IX Encuentro Nacional de Experiencias en Enseñanza de la Biología y la Educación Ambiental. IV Congreso Nacional de Investigación en Enseñanza de la Biología.

La adopción de un modelo de ciencia y de enseñanza por parte del profesor de ciencia.

\begin{tabular}{|c|c|c|}
\hline Valoración & Número & Porcentaje \\
\hline Totalmente de Acuerdo & 9 & $53 \%$ \\
\hline Parcialmente de Acuerdo & 7 & $41 \%$ \\
\hline Parcialmente en Desacuerdo & 1 & $6 \%$ \\
\hline Totalmente en Desacuerdo & 0 & $0 \%$ \\
\hline Total & 17 & $100 \%$ \\
\hline
\end{tabular}

El conocimiento que se produce en la comunidad científica es verdadero, confiable, definitivo e incuestionable. El $59 \%$ de los docentes están de acuerdo sobre la verdad y confiabilidad del conocimiento científico, que refleja una mirada absolutista que encaja en una de las características del modelo tecnológico (Guisasola \& Morentin, 2007), cuadro 5.

Cuadro 5:

El conocimiento que se produce en la comunidad científica es verdadero, confiable, definitivo e incuestionable.

\begin{tabular}{|c|c|c|}
\hline Valoración & Número & Porcentaje \\
\hline Totalmente de Acuerdo & 1 & $6 \%$ \\
\hline Parcialmente de Acuerdo & 9 & $53 \%$ \\
\hline Parcialmente en Desacuerdo & 4 & $24 \%$ \\
\hline Totalmente en Desacuerdo & 3 & $18 \%$ \\
\hline Total & 17 & $100 \%$ \\
\hline
\end{tabular}

El cambio de una teoría científica por otra se basa en criterios objetivos: prevalece la que explica mejor el conjunto de fenómenos a que se refiere. El $94 \%$ de las docentes encuestadas están de acuerdo sobre la objetividad de las teorías científicas que refleja una visión acumulativa y considera un crecimiento lineal del conocimiento científico, dejando de lado las crisis y remodelaciones profundas de las teorías científicas (Gil et al, 2001), cuadro 6.

Cuadro 6:

El cambio de una teoría científica por otra se basa en criterios objetivos: prevalece la que explica mejor el conjunto de fenómenos a que se refiere. 
Bio - grafía. Escritos sobre la Biología y su Enseñanza. ISSN 2027-1034

Edición Extraordinaria. p.p. 1325 - 1332

Memorias del IX Encuentro Nacional de Experiencias en Enseñanza de la Biología y la Educación Ambiental. IV Congreso Nacional de Investigación en Enseñanza de la Biología.

\begin{tabular}{|c|c|c|}
\hline Valoración & Número & Porcentaje \\
\hline Totalmente de Acuerdo & 8 & $47 \%$ \\
\hline Parcialmente de Acuerdo & 8 & $47 \%$ \\
\hline Parcialmente en Desacuerdo & 0 & $0 \%$ \\
\hline Totalmente en Desacuerdo & 1 & $6 \%$ \\
\hline Total & 17 & $100 \%$ \\
\hline
\end{tabular}

Difícilmente cambian los conocimientos científicos que han adquirido un reconocimiento y legitimación universal. El $52 \%$ de los docentes encuestados consideran que los conocimientos científicos que han tenido aceptación en la comunidad científica difícilmente se cambian. Consideran que la ciencia es estática y única y difícilmente cambia a través del tiempo, Cuadro 7.

Cuadro 7:

Cambios en los conocimientos científicos que tienen reconocimiento y legitimación universal.

\begin{tabular}{|c|c|c|}
\hline Valoración & Número & Porcentaje \\
\hline Totalmente de Acuerdo & 1 & $6 \%$ \\
\hline Parcialmente de Acuerdo & 8 & $47 \%$ \\
\hline Parcialmente en Desacuerdo & 4 & $24 \%$ \\
\hline Totalmente en Desacuerdo & 4 & $24 \%$ \\
\hline Total & 17 & $100 \%$ \\
\hline
\end{tabular}

\section{CONCLUSIONES}

En las docentes de primaria, coexisten diversas representaciones acerca de la naturaleza de la ciencia, transitan entre una concepción constructivista y una concepción dogmática.

El nivel de comprensión sobre naturaleza de la Ciencia de los docentes está en un plano procedimental y corresponde a una representación de NdC empíricapositivista. Al momento de enseñar ciencias, se prioriza la observación y la experimentación, porque permitirían al estudiante llegar a descubrir por sí mismo las leyes de la naturaleza (Pro Bueno, 2001). Pero en la realidad, no se utilizan muchas actividades experimentales en las aulas. 
Bio - grafía. Escritos sobre la Biología y su Enseñanza. ISSN 2027-1034

Edición Extraordinaria. p.p. 1325 - 1332

Memorias del IX Encuentro Nacional de Experiencias en Enseñanza de la Biología y la Educación Ambiental. IV Congreso Nacional de Investigación en Enseñanza de la Biología.

Esta imagen empírica de la ciencia, no se aleja de otras concepciones docentes cuya diversidad epistemológica se relacionan con el racionalismo, relativismo y empirismo (Martínez, 2001), siendo el empirismo la tendencia más frecuente en los docentes de primaria, que sigue siendo una concepción ampliamente extendida entre el profesorado de ciencias (Fernández, 2002).

Según la red conceptual para la dimensión naturaleza de la ciencia los profesores de primaria asignan mayor importancia a los aspectos procedimentales y conceptuales. Prevalece una noción empirista de la construcción del conocimiento y el escenario de la actividad científica escolar es rígido, inflexible y pobremente evolutivo.

\section{REFERENCIAS}

Acevedo, J. (2008). El estado actual de la naturaleza de la ciencia en la didáctica de las ciencias. En: revista eureka sobre enseñanza y divulgación de las ciencias, vol. 5. No 2, p. 134-169.

Arrieta, J. y Daza, S. (2014). Representaciones mentales sobre la naturaleza de las ciencias en docentes de las áreas profesionalizantes del programa de Ingeniería Agronómica de UNIPAZ. Tesis de maestría en educación. Bucaramanga, universidad autónoma de Bucaramanga. Colombia, p. 16-258.

Fernández, I., Gil, D., Carrascosa, J., Cachapuz, A., \& Praia, J. (2002). Visiones deformadas de la ciencia transmitidas por la enseñanza. Enseñanza de las ciencias, 20(3), 477-488.

Gil, D., Fernández, I. \& Carrascosa, j. (2001). Hacia una imagen no deformada de la actividad científica. Endoxa: series filosóficas, vol. 1, no 14, p. 228-260.

Guisasola, J. Morentin, M. ¿Comprenden la naturaleza de la ciencia los futuros maestros y maestras de educación primaria? Reec: en: revista electrónica de enseñanza de las ciencias, vol. 6, no 2. 2007, p. 246-262. 
Bio - grafía. Escritos sobre la Biología y su Enseñanza. ISSN 2027-1034

Edición Extraordinaria. p.p. 1325 - 1332

Memorias del IX Encuentro Nacional de Experiencias en Enseñanza de la Biología y

la Educación Ambiental. IV Congreso Nacional de Investigación en Enseñanza de la Biología.

Porlan, R., Martín del pozo, R. (1996). Ciencia, profesores y enseñanza: unas relaciones complejas. Alambique, 8 (2), pp. 23-32.

De pro Bueno, a. J. (2003). La construcción del conocimiento científico y los contenidos de ciencias. In enseñar ciencias (pp. 33-54). Graó.

Pujalte, P., Bonan, L., Porro, S. \& Aduriz-Bravo, A. (2014). Las imágenes inadecuadas deficiencia y de científico como foco de la naturaleza de la ciencia: estado del arte y cuestiones pendientes. Ciencia \& educação, bauru, 20(3), 535-548.

Quintanilla, m., Labarrere, a., Santos, m., Cadiz, j. Cuéllar, I.,Saffer, g. \& Camacho, j. (2005). Elaboración validación y aplicación preliminar de un cuestionario sobre ideas acerca de la imagen de ciencia y educación científica de profesores en servicio, grupo Grecia departamento de didáctica. Facultad de educaciónpontificia universidad católica de chile. $25 \mathrm{p}$. http://www7.uc.cl/sw_educ/educacion/grecia/plano/htm//pdfs/linea_investigacion/ que_ciencia_ensenar_iec/iec_045.pdf

Quintanilla, M., \& Ravanal., E., (2009). Racionalidades epistemológicas y didácticas del profesorado de biología en activo sobre la enseñanza y aprendizaje del metabolismo: aportes para el debate de una nueva clase de ciencias (Doctoral dissertation, Universidad Academia de Humanismo Cristiano).

Tamayo, E, Sánchez, B., Buriticá, A., \& Clemencia, O. (2010). Concepciones de naturaleza de la ciencia en profesores de educación básica. Revista latinoamericana de estudios educativos (1900-9895), 6(1).

Wang, h. A., Marsh, d. D. (2002). Science instruction with a humanistic twist: teachers' perception and practice in using the history of science in their classrooms. Science \& education, 11(2), 169-189. 